\title{
STS: A Real Time Scheduling Algorithm for Temporal-Spatial Tasks in Wireless Networked Control Systems
}

\author{
Qiang Lin $^{1,2, *}$, Guowei Wu ${ }^{1}$ Qing Liu ${ }^{1}$ Zhaowei Huang ${ }^{1}$ \\ ${ }^{1}$ School of Software Technology, Dalian University of Technology, Dalian \\ 116023, China \\ ${ }^{2}$ Dalian Institute of Science and Technology, Dalian 116052, China \\ * Corresponding author. Email address: lqchina@126.com (Qiang Lin)
}

\begin{abstract}
In this paper, a hybrid spatial and temporal scheduling algorithm for wireless networked control system, which takes the distance between robot and task region as well as the deadline of a task into consideration is proposed. In our method, we quantify spatial and temporal factors for eventually getting the priority queue. Based on the priority queue, the robot is able to execute the tasks sequentially. At last, extensive simulations are conducted to show the advantages of our spatial and temporal scheduling algorithm. Simulation results show that, on the premise of ensuring high successful scheduling rate, compared with previous method, our scheme show merits in terms of task requesting throughput, successful task solving ratio, average responding delay.
\end{abstract}

\section{Introduction}

Wireless Networks Control Systems (WNCSs) use the wireless communication network to realize information exchange between the geographically distributed and portable field sensor, the controller and the actuator, in order to get real-time feedback from the controlled system [1, 2, 3]. As a closed loop control system instead of the traditional point-to-point connections, it breaks the traditional limitation in the physical space and expands the control activities. At the same time, it reduces the complexity of connection in the system and improves the information integration. The WNCS has many advantages, such as resource sharing, high diagnostic capacity, high mobility, the reduction in system wiring, the increase in flexibility and robustness, the convenience of installation and maintenance. WNCS is usually regarded as the safety (even life)-critical system, which requires to monitor and control the physical world safely, reliably, eff ectively and in real time. Therefore, WNCS demands high reliability in mandatory. Researchers have pointed out that: one of the primary questions to build up highly reliable WNCS is to ensure its real-time and reliability strictly.

Real-time scheduling is a key method and an eff ective way of ensuring the real-time and reliability of the WNCS system. Although there have been numerous progresses and studies on the real-time scheduling of the networked controlling system in the last decade, the real-time scheduling is still an open question so far. Some features, such as systematic dynamicity and strict constraints of resources of WNCS bring great challenges to the real-time scheduling of WNCS, which involves multiple mutual-related targets (e.g. reliability, real-time and controlling quality etc.) as well as the interdependent decision variable (such as transmission scheduling, routing, and sampling rate etc.). The detailed challenges indicate (but are not limited to) the following:

1. Random propagation delay and packet loss. As the wireless network transmission form is multi-hoping, great randomness will exist in the progress of the propagation delay and packet loss, which increase the rate of data packet loss in the wireless network system [4].

2. Communication interruption. Due to network nodes have good mobility, network 
topology is dynamic. And the high rate of packet loss will lead to intermittent connection in the process of communication, sometimes there will be a long time communication interruption [5].

3. Time-varying channel gain and interference. Time-varying channel gain is due to the wireless channel varies with time and space. At the same time, there is interference between each node [6].

4. Bandwidth is relatively small, making the transmission power is limited and the bit error rate keeps high [7].

Since a WNCS is widely used in real life, it has been used in wireless remote robot control system, wireless smart home network control system, wireless vehicle control system and so on. So far, outstanding contributions have been made in various fields of social life, such as industrial control, medical and military, especially in today's smart house using smart phones as the core to implement a control of the smart home wireless network. A complete set of wireless network control system can run as a support to ensure its normal work $[8,9]$.

The rest of this paper is organized as follows. In Section 2, we give detailed information of the proposed scheme. Section 3 performs extensive simulations to show the performance of our scheme. Finally, we conclude this paper in Section 4.

\section{Our Scheme}

In this section, we will detail the space-time hybrid priority scheduling algorithm. For a wireless mobile robot, choosing a task request from service pool means a clear queuing behavior. It encourages us to adopt a queuing model to research and analyze the scheduling process: the mobile robot runs as "server" and the task request sent by the node is referred to as "client".

\subsection{Related Symbols and Definitions}

In this section, related symbols and corresponding definitions are given below.

Table 1. Symbols and Definitions

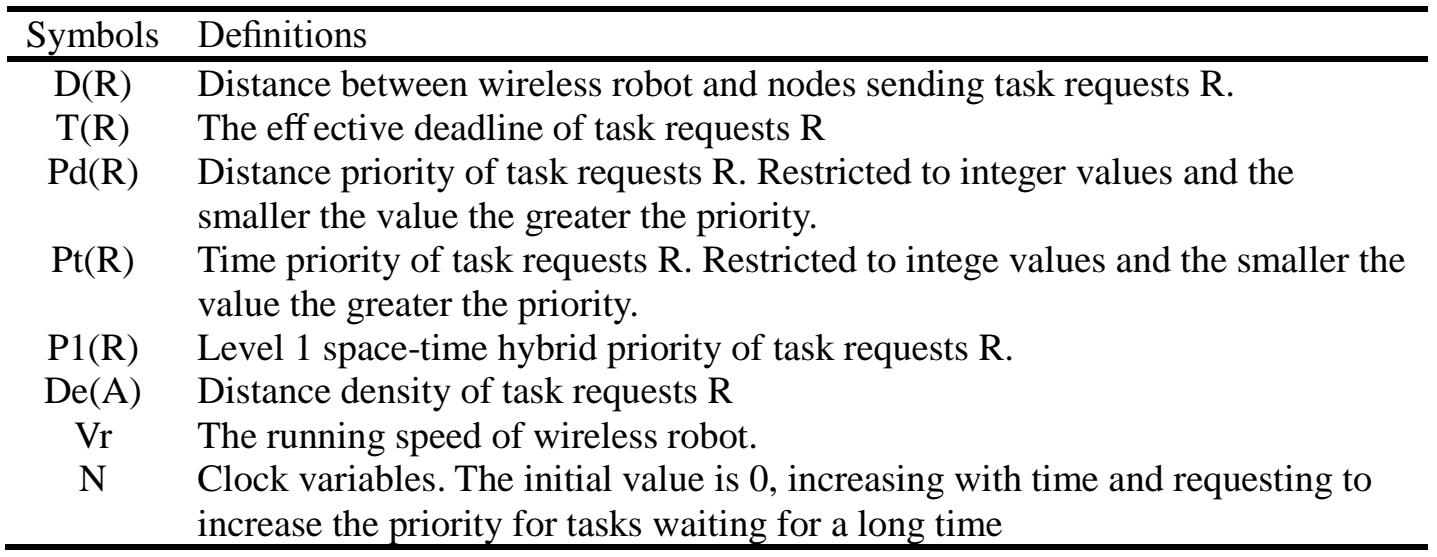

\subsection{Problem Definitions}

Figure 2 depicts the framework of on-demand task in the progress of request and response. In our scenario, we deploy a set of nodes in an area with side length $\mathrm{L}$. When a node initialize a demand, it will immediately send a task request to the wireless robot who will immediately response. In addition, we assume that sensor nodes can locate the wireless robot in real time [10]. 


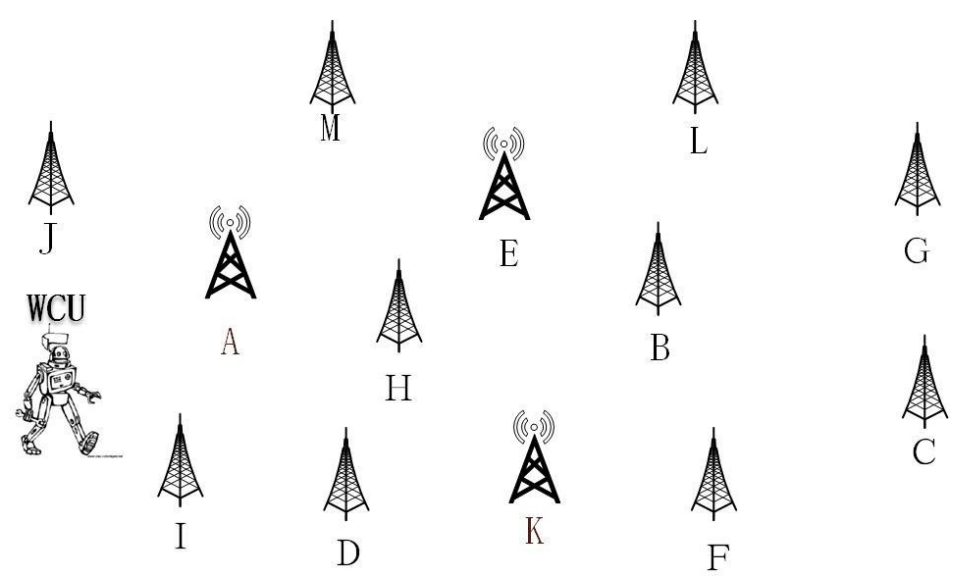

\section{Current Service Pool: A K E \\ Node with request. Node without request.}

Figure 1. System Architecture

Similarly, in this paper we only consider the situation that there is only one available wireless robot in the task response sensor network [11, 12,13,14]. Wireless robot collects all requests and keeps these requests in the service pool. As showed in figure 2, the service pool records the location information of node A, K, E. Because task request is constrained by the real time (Being constrained by the real time means that the task request has real-time tasks eff ective deadline), task request scheduling problem can be thought as a real-time task scheduling problem. This requires us to queue the task requests according to the urgency and priority. Once a request is selected as a node to be responded immediately, the wireless robot will solve the task requests for it immediately. As a result, the robot's travel path is directly associated with the order of the tasks in the queue. In the on-demand frame, wireless robots run at a constant speed. The demand time to solve the task is too short when compared with the travel time, so it will be ignored in this article.

\subsection{Infrastructure of Algorithm}

Both of the movements made by the wireless robot, marching to the corresponding node and responding task of node, have relationships with the time and space, so we should balance them in the scheduling algorithm $[15,16,17,18]$. In this algorithm, the time priority and space priority are used at the same time to confirm the final mixed priority, for guiding the robot to response to the task of node.

Set $D(R)$ as the distance variable from the task request node to the robot. Use this algorithm to calculate the priority value of nodes' task requests. On-demand scheduling must meet the different requirements, such as: the independency of time and space, preemption, priority adjustment and so on. Then we'll introduce some corresponding methods to meet the above requirements.

\subsection{The Determination of Tasks Eff ectiveness Deadline}

Space-time hybrid priority scheduling algorithm (STS) has a task eff ective deadline. Here we will illustrate how to calculate the task eff ectiveness deadline T firstly. Define L and $\mathrm{V} \mathrm{r}$ to respectively represent the side length of the wireless sensor network and the running speed of wireless robot. Tm represents the maximum time consumed from one 
location to another within the square area. We can get such a formula (1).

$$
T m=\sqrt{2} * L V r
$$

In order to ensure all nodes can get responses from the robot before tasks fail, the task eff ective deadline of the node should be at least Tm. The waiting queue length Lq of task response nodes must be taken into consideration, because not all the task requests can be immediately responded. Therefore, tasks eff ectiveness deadline $\mathrm{T}$ can be expressed in the following formula (2)

$$
T=T m * L q
$$

\subsection{Preemption}

Here we analyze the case that preemption exists in scheduling. Because new task request may appear in the service pool at any time and the new one may be higher in time and space priority than the existing one in the service pool, which will lead to a few task requests always be preempted and never get responded [19]. To solve this problem, a clock variable $\mathrm{N}$ is added in each task request in this paper. Set the initial value of $\mathrm{N}$ as 0 . With the increasing of time, we multiply the appropriate coefficient in $\mathrm{N}$ and add the value to the current priority value to make the task having waited for a long time get a higher priority and avoid preemption.

\subsection{Space-time Hybrid Priority Scheduling Algorithm (STS)}

In this section, we introduce how to compare the priority of two task requests in detail. There are two factors introduced in this paper, which decide the priority of task request. One is the time factor, namely the task request eff ectiveness deadline, the shorter the task eff ectiveness deadline the higher the time priority. Another is the space factor, namely the distance from the node sending task requests to the wireless robot, the closer the distance the higher the distance priority.

First of all, we compare the time priority of the two task requests. Compare the eff ectiveness deadline of the two task requests according to the above algorithm and get the time priority values of task requests. The greater the time priority the topper the location in time priority queue, namely the position number is smaller in the queue.

The temporal priority of a request $A$ is bigger than that of $B$ iff $T(A)<T(B)$, otherwise, $\operatorname{Pt}(\mathrm{A})>\operatorname{Pt}(\mathrm{B})$. Next we compare the distance priority of the two task requests

The spatial priority of a request $A$ is bigger than that of $B$, iff $D(A)<D(B)$, otherwise, $\operatorname{Pd}(\mathrm{A})>\operatorname{Pd}(\mathrm{B})$.

We compare distance priority of the two task requests. The greater the distance priority the topper the position in the distance priority queue, namely the position number is smaller in the queue.

Then, we can get two priority queues formed from task requests in the service pool. It has been put forward in the front part of the paper that the time and distance priority values are integer, and the smaller the value the higher the priority. Therefore, the author set the position values of the time priority queue as the time priority values, the position values of the space priority queue as the space priority values. There is a level 1 space-time hybrid priority position for each task request, we can preliminarily determine scheduling order of wireless robots task responses. $\mathrm{Pt}(\mathrm{A})$ and $\mathrm{Pd}(\mathrm{A})$ respectively represent time priority and space priority of the task request A. Values are respectively the position number of task request $\mathrm{A}$ in the time and the space priority scheduling queue. We calculated values of level 1 space-time hybrid priority P1(A) by formula (3) and obtain the scheduling order of task request preliminary.

$$
P 1(A)=\gamma P t(A)+\lambda P d(A)
$$

where, $0 \leq \lambda \leq 1,0 \leq \gamma \leq 1 . \gamma$ and $\lambda$ respectively emphasize the proportion of 
distance priority and time priority in the process of scheduling. If $\gamma \lambda \rightarrow \infty$, the algorithm is the earliest deadline first principle EDF. On the contrary if $\lambda \rightarrow \infty$, the algorithm becomes the shortest distance preemption principle (EDF. In this paper, we set $\gamma \lambda=1$.

After getting level 1 space-time hybrid priority, there would be two task requests having equal value in level 1 space-time hybrid priority when multiple node tasks are in the thread pool, as the points on the diagonal showed in figure 3 . However, it is not likely for wireless robot scheduling in response to the two task request nodes at the same time. So we bring up the second step in the space-time hybrid priority scheduling algorithm to determine the order of two nodes which have equal level 1 space-time hybrid priority. When two requests' hybrid priority is equivalent to each other, we compare the value of De(A). A higher value leads to a bigger priority. Hence, the comparison between two requests is completed

Here we introduce the concept of distance density $(\operatorname{De}(A))$. Firstly the smaller the value of distance density, the higher the priority of the task request. Distance density referenced the value concept in [8]. We can get the formula(4).

$$
D e(A)=D(A) * T(A)
$$

At the last, we order the task requests in the service pool according to the level 2 priority rules in algorithm 4. Let wireless robot to response task and solve the task requirements according to the order of corresponding node. But before solving node task requests, the values have to be inspected in formula (5).

$$
V=\frac{D(A)}{T(A)}
$$

If $V<V \mathrm{r}$ or $V=V r$, it means wireless robots have the ability to reach nodes to solute task requests before requests fail, so they will reach the node and solve task requests immediately. And if $V>V r$ wireless robot cant arrive at the node to solute task requests before requests fail, so wireless robot will delete the task request node from service pool and the node will become bad. This will help avoiding the situation that the node has become bad before wireless robots reach the corresponding node, so as to avoid the waste of travel time and delay of the whole scheduling process.

\section{Simulations and Results Analysis}

\subsection{Simulation Setup}

Table 2. Simulation Parameters

\begin{tabular}{ll}
\hline parameter & value \\
\hline Size of wireless sensor network area & $100 \mathrm{~m} 100 \mathrm{~m}$ \\
Number of wireless sensor nodes & 100 \\
Running speed of wireless robot $\mathrm{Vr}$ & $2 \mathrm{~m} / \mathrm{s}$ \\
\hline
\end{tabular}

As showed in table 2, in the simulated experiment, the author randomly places 100 nodes in a $100 \mathrm{~m}$ by $100 \mathrm{~m}$ square area. Set the running speed of wireless robot as $2 \mathrm{~m} / \mathrm{s}$. Wireless robot will use the above algorithm to sort all the task requests in service pool and then solve task requests from the current position to the location of the target node according to the waiting queue order.

\subsection{Throughput}

In the on-demand architecture, throughput of task requests is seen as an important assessment factor of the scheduling algorithm. For wireless mobile robot, the throughput in the scheduling process is defined as the number of task requests that a mobile robot can solve in a certain period of time. It is an important measure to assess the ability of a system to provide task response services for each node. In general, a higher system 
throughput means the shorter processing time of wireless mobile robot, which in turn means lower consumption of a mobile robot.

For mobile robot, choosing requests from the service pool means a clear queuing behavior, it encourages us to adopt a queuing model to research and analyze the scheduling process: mobile robot run as the "server", task requests sent by the node are seen as the "client". We have mentioned above that an M/M/1/EDF queuing model is adopted in the paper; here we don't take it again.

From the figure 4 we can see that the throughput in the space-time hybrid priority scheduling algorithm STS is growing gradually as the growth of the time. However, the throughput of the shortest distance priority preemption scheduling algorithm EDF is not stable. The throughput of STS is much larger than EDF, sometimes the latter is only a little more than half of the former. It can be seen clearly that the task responses scheduling efficiency has a huge improvement in space-time hybrid priority scheduling algorithm.

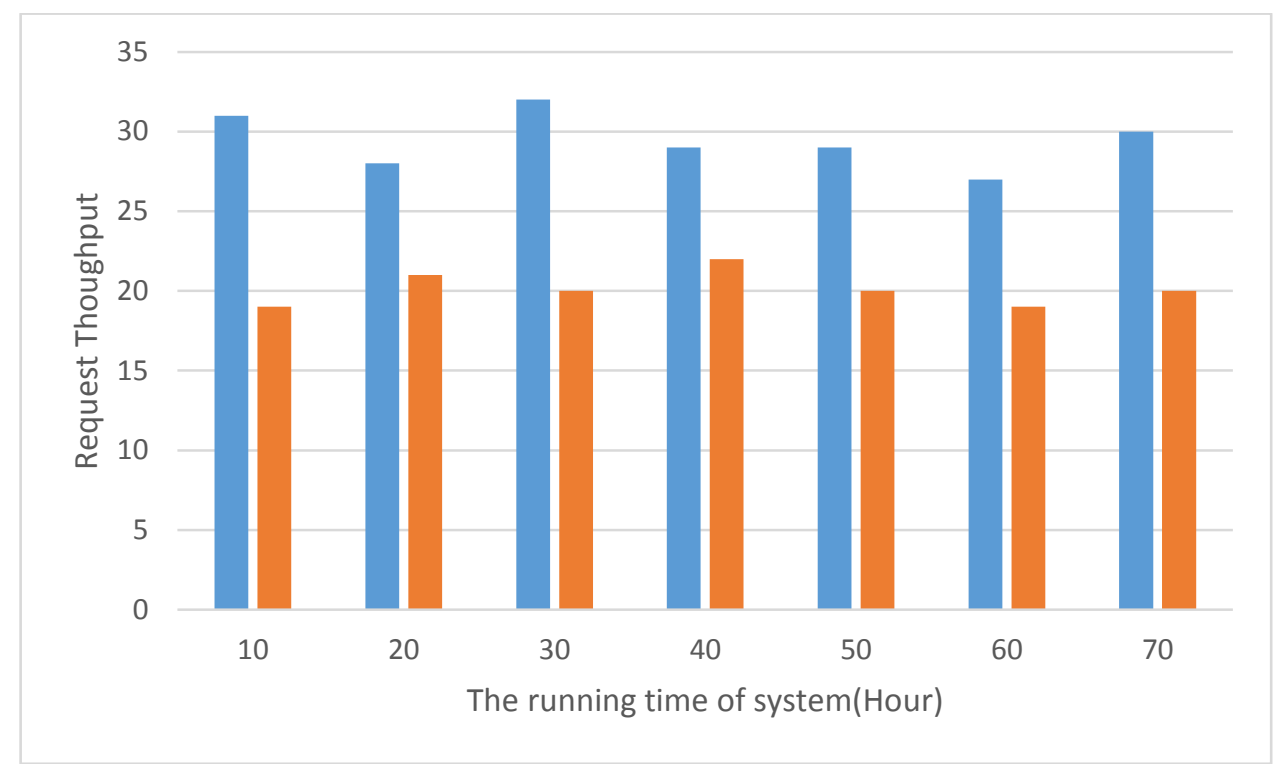

Figure 2. Comparison of Throughput

\subsection{Average Response Time}

In this part, the author calculates the average response time. It refers to the time starts from the moment that sensor nodes make requests and stops at the moment that wireless robot receives request and comes to solve task requests. It reflects task response speed and scheduling efficiency problem of the wireless robot and it is also an important measure of the algorithm.

Figure 2 shows that the average response time of STS slows down from the overall, while the EDF is falling fast. This is because under the EDF, the task request in the wireless sensor network nodes has not been resolved timely, leading to failure of nodes and decline of nodes number. Even so, the average response time of EDF is slower than STS for nearly five minutes at last. The shorter response time means the shorter nodes waiting time, this improves the chances that nodes task requests can get solved and indicates the efficiency of task response and solution in STS is higher than that in EDF. 


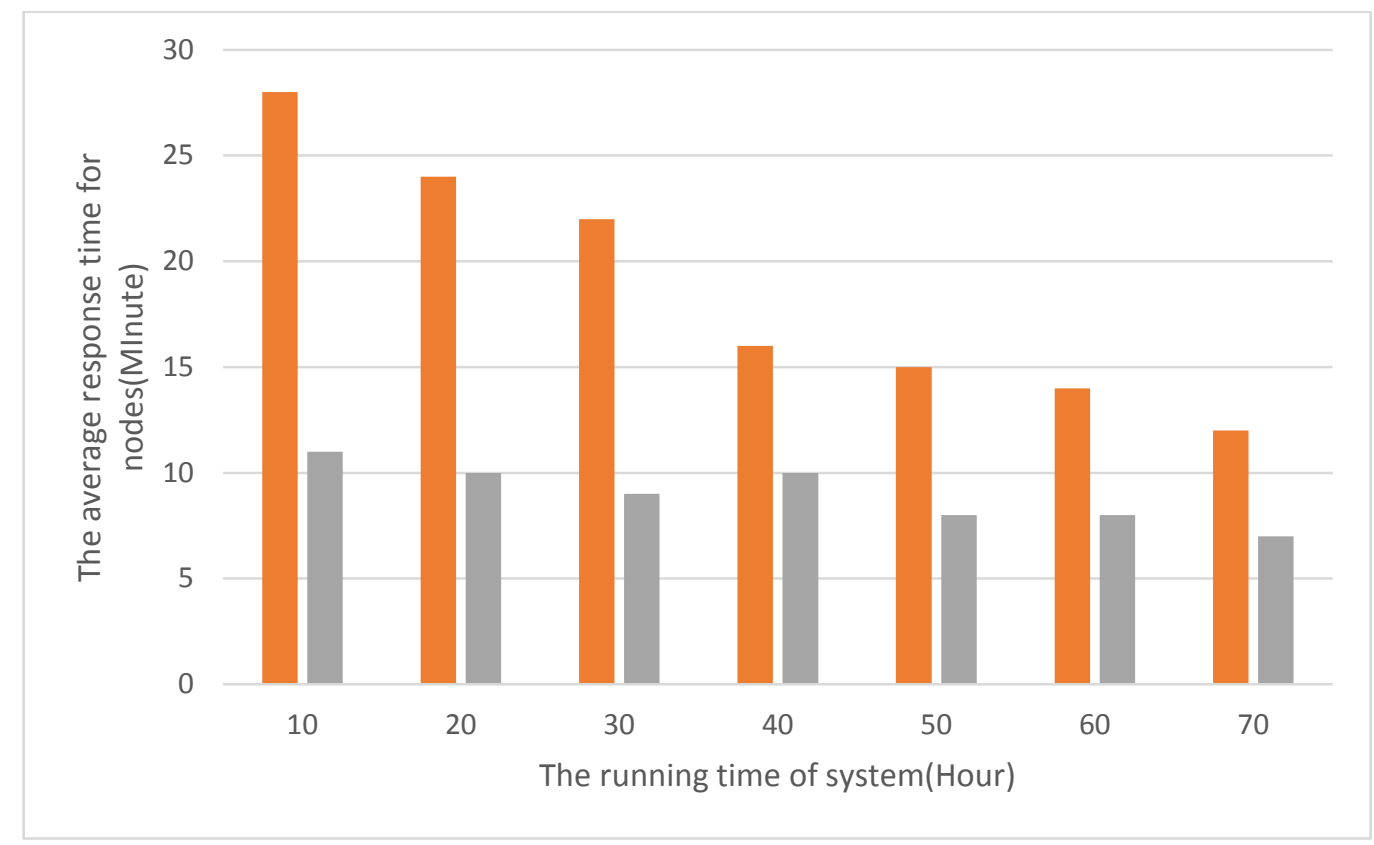

Figure 3. Comparison of Average Response Time

\subsection{Average Length of the Waiting Queue}

In this part, we measure the average length of task requests' waiting queue. The average length of the waiting queue is the number of task requests in the waiting queue per second. This factor is used to show the wireless robots working condition and sensor nodes waiting condition.

From figure 3, we can clearly see that the average length of the waiting queue in STS is always stable at around 4, while the value in EDF is far larger than the former. A shorter waiting queue length means a faster rate of the completion of task nodes and a more continuous working condition of nodes. This fully shows the high efficiency of wireless robot, which also proved the superiority of the algorithm.

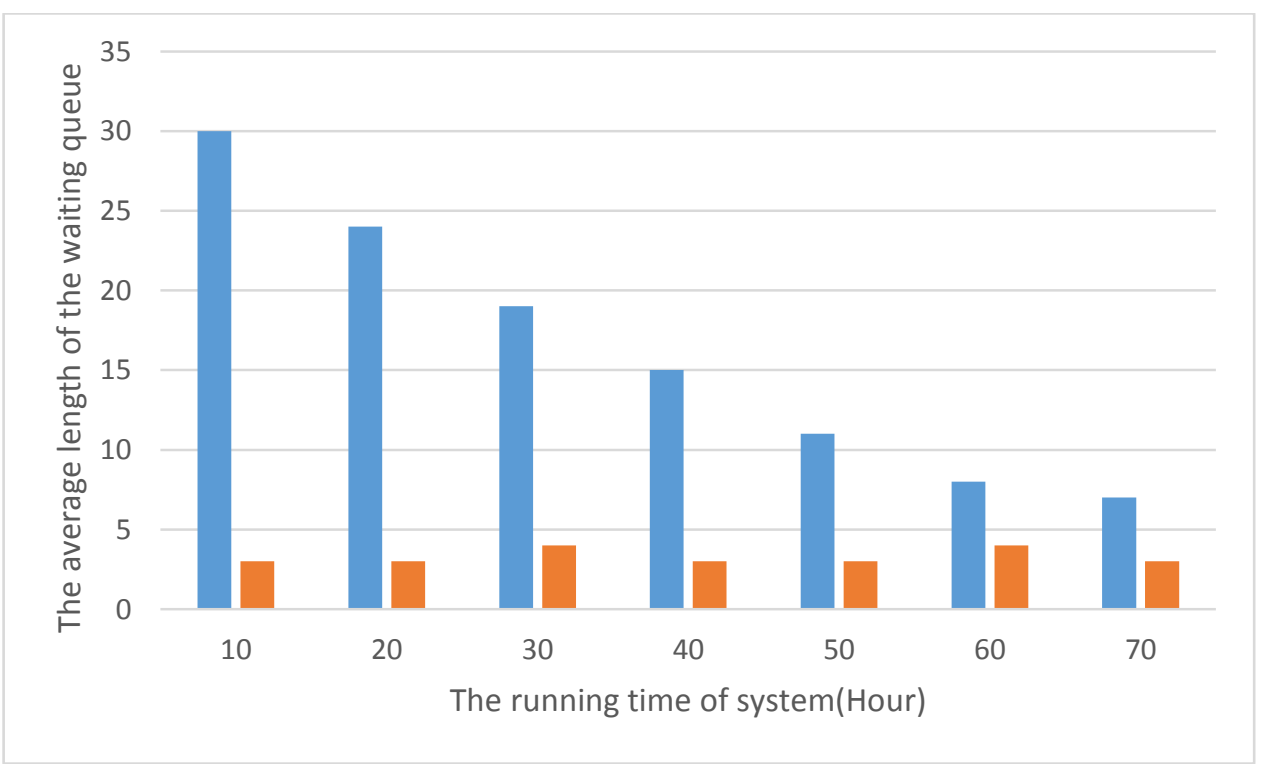

Figure 4. Comparison of Waiting Queue's Length 


\subsection{Success Rate}

The author compares the task solutions success rate of wireless robots under the STS and EDF in the following part. As showed in figure 4, though the success rate that wireless robot to solve tasks is increasing no matter in the STS or EDF, the former is far higher than the latter. The reason success rate under the EDF increase is that sensor tasks didn't get timely solved leading to the number of eff ective nodes decrease. The declining of the number of nodes makes the success rate get higher. While the success rate in task solution always keeps high in STS.

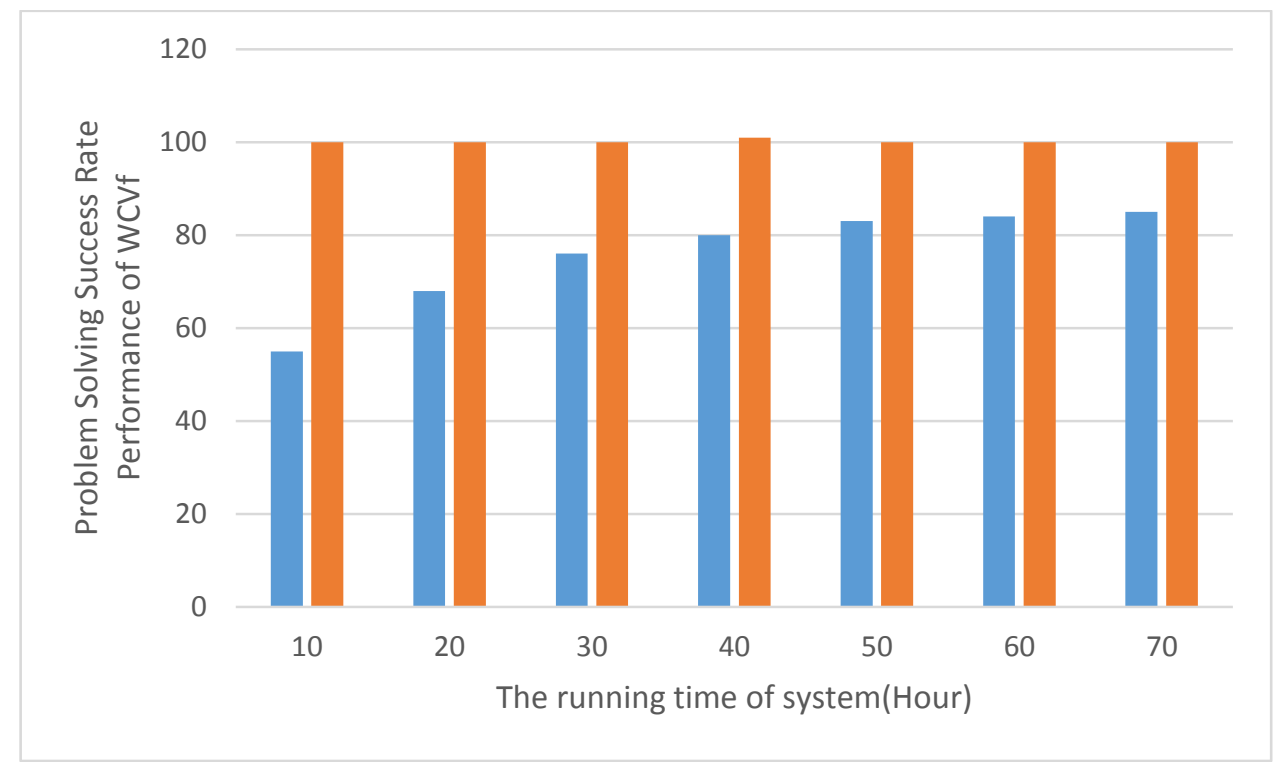

Figure 5. Comparison of Success Rate in Task Solution

This indicates that the wireless robot can basically meet the task request of sensor nodes and eff ectively complete the node's task. Therefore, we can conclude the STS can improve the success rate in the task solution of the wireless robot.

\subsection{Average service time}

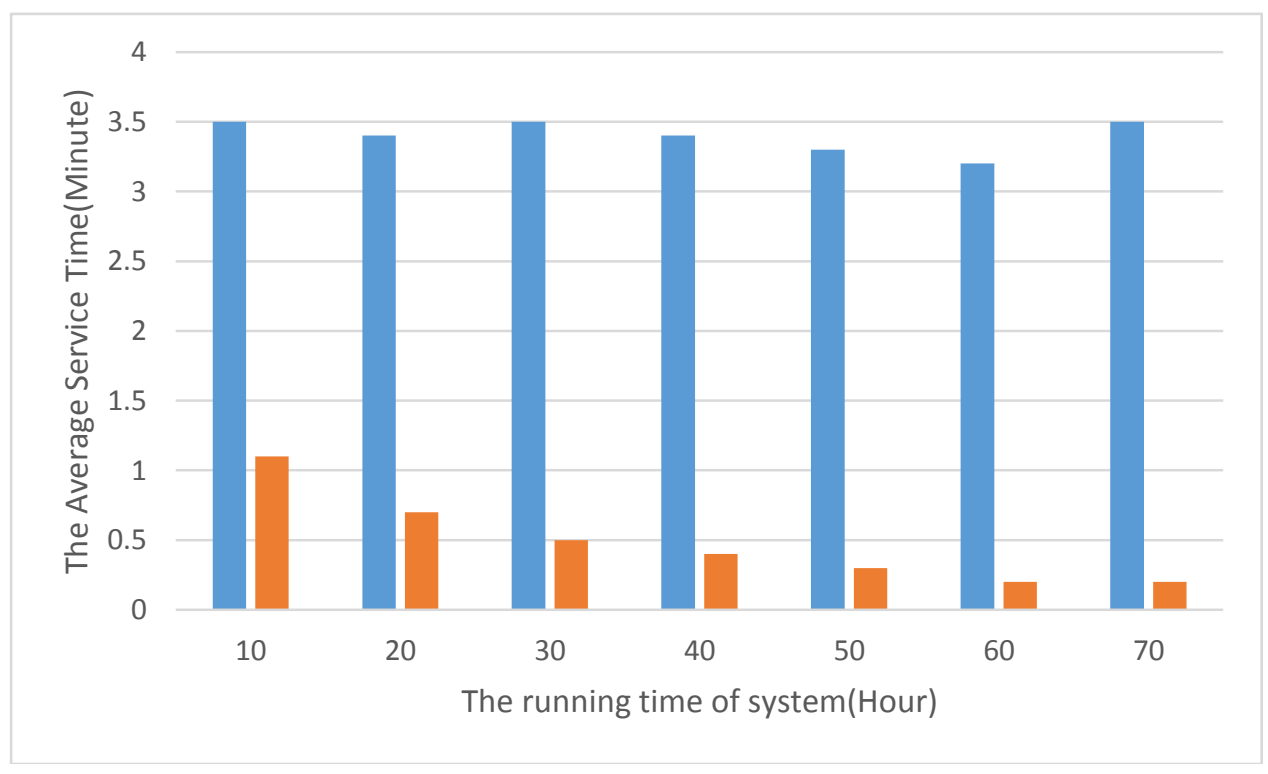

Figure 6. Comparison of the Average Service Time 
Finally, the author compared the average service time of the two kinds of algorithm. The average service time refers to the average time spent by the wireless robot in the process of solving each task request. Average service time can explain the efficiency under the two algorithm STS and EDF in a certain range.

As showed in figure 6, the average service time of the wireless robot under EDF is 4 to 5 times than that of the STS obviously. In other words, the wireless robot under EDF will spend more 3 minutes in the same condition than that under STS every time when it completes a task. So we can come to the conclusion that the wireless robot's planning path under STS has got optimized greatly. A large number of tasks can get responses in a very short time, so the improvement of task solution's efficiency is significant.

The average service time should be consisted from two parts: the travel time spent by the wireless robot from the current position to the location of task node and the consumed time to solve tasks for nodes. In practice, the time consumed by the latter is quite lower compared with the former, so it won't aff ect the result of the experiment at all. And the main purpose of this paper is to compare the advantages and disadvantages of these two algorithms, so we do not consider the factor in the experiment. In this paper, the average service time mentioned above refers to the travel time spent by the wireless robot from current position to the location of task node.

\section{Conclusions}

In this paper, we proposed a scheduling algorithm for on-demand architecture of wireless sensor network (WSN), space-time hybrid priority scheduling algorithm (STS). In the STS, we respectively get a distance queue and a task eff ectiveness deadline queue from the time factor and the space factor. Then we calculate the two queues preliminary and get an original task scheduling order. In the next step, the concept of distance density is put forward to identify the scheduling order of task requests which have the same level hybrid priority according to the distance density and clock variables. At the last, we get the final order of the whole task scheduling. It is worth to mention that the time and space factors are considered at the same time in this paper, avoiding the extreme problem that happens when they are considered separately. In the latter part of the paper, we conduct a serious of simulation experiments and carry out performance evaluation to the algorithm. By analyzing the data in experimental results, we prove the eff ectiveness of the proposed algorithm. The experimental results show that the performance of the new algorithm in throughput, success rate of task solution, average service time and so on, is better than EDF.

In this paper, we just consider that there is only one wireless robot in the wireless sensor networks (WSNs). However, in reality, it is obviously impossible to arrange a single wireless robot to solve tasks for all the nodes in large-scale wireless sensor networks. Therefore, in the future work, the author will commit in the research that there are multiple wireless robots being in collaboration to solve tasks for the sensor nodes in the networks. And then the task solution efficiency of wireless robot will improve significantly.

As part of our future works, we will pay close attention to use STS algorithm in real world.

\section{Acknowledgement}

This research is sponsored in part by the National Natural Science Foundation of China (No.61173179 and No.61202441), Nuclear high base of major projects (2012ZX01039-004-21) and Program for New Century Excellent Talents in University (NCET-13-0083). 


\section{References}

[1] M. Pajic,S. Sundaram,G.J. Pappas et al.The Wireless Control Network: A New Approach for Control Over Networks[J]. IEEE Transactions on Automatic Control,2011,56(10):2305.

[2] Y.A. Millian, F. Vargas, F. Molona et al.A Wireless Networked Control Systems review:Robotics Symposium, 2011 IEEE IX Latin American and IEEE Colombian Conference on Automatic Control and Industry Applications (LARC),2011[C]. Bogota: IEEE ,2011: 1C6.

[3] J.H. Taylor,H.M.S. Ibrahim. A new, practical approach to maintaining an efficient yet acceptablyperforming wireless networked control system: System Science and Engineering (ICSSE), 2010[C]. Taipei: IEEE,2010: 269 C 274.

[4] P. Ferrari,A. Flammini,M. Rizzi et al. Improving simulation of wireless networked control systems based on WirelessHART[J].Computer Standards \& Interfaces,2013,35(6): 605.

[5] U. Tiber,C. Fischione,K.H. Johansson et all.Energy-efficient sampling of networked control systems over IEEE 802.15.4 wireless networks[J].Automatica,2012,49(3):71

[6] Wang Gang.Wireless remote controltechnology in intelligent buildings system: World Automation Congress (WAC), 2012[C]. Puerto Vallarta, Mexico: IEEE,2012:231-233.

[7] Gao Yudong,Wu Jinjie,Hu Min et al. Design and Development of Hardware-in-Loop Simulation of Spacecraft Attitude Control System Based on Wireless Ad Hoc Networking: Industrial Control and Electronics Engineering (ICICEE), 2012[C]. Xi'an : IEEE,2012:584-587.

[8] Gurkan Tuna,V. Cagri Gungor,Kayhan Gulez et al. An autonomous wireless sensor network deployment system using mobile robots for human existence detection in case of disasters[J]. Ad Hoc Networks,2012,13(A):54.

[9] Ma Xinhua,Rong Xiaofeng.Real-time performance analysis of wireless sensor networks based on queuing model[J].Journal of Xi' an University of Posts and Telecommunications,2009,14(1):112.

[10] Xinhua Ma, Xiaofeng Rong. Analysis of real-time performance for wireless sensor networks based on queuing theory[J]. Journal of Xi' an University of post and telecommunications, 2009,14(1):112.

[11] $\mathrm{Xu} \mathrm{Li,R}$. Falcon,A. Nayak et al.Servicing wireless sensor networks by mobile robotsJ. Communications Magazine, IEEE, 201250(7):147.

[12] Xia Jiali,Chen Hui,Yang Bing. A dynamic priority real-time task scheduling algorithm[J]. Chinese Journal of Computers, 2012,35(12):2685.

[13] Jiali Xia, Hui Chen, Bing Yang. A Real-Time Tasks Scheduling Algorithm Basd on Dynamic Priority[J]. Chinese Journal of Computers, 2012,35(12):2685.

[14] Xie Liguang, Shi Yi,Y. Thomas Hou et al. On Traveling Path and Related Problems for A Mobile Station in A Rechargeable Sensor Network: 14th ACM international symposium on Mobile ad hoc networking and computing (Mobihoc 2013),2013C. New York :ACM New York,2013:109-118.

[15] Dai Haipeng, Wu Xiaobing,Xu Lijie et al.Practical Scheduling for Stochastic Event Capture in Wireless Rechargeable Sensor Networks: Wireless Communications and Networking Conference (WCNC 2013),2013C. Shanghai : IEEE ,2013:986-991.

[16] Wang Yongyan,Wang Qiang,Wang Hongan et al.A Real-Time Scheduling Algorithm Based on Priority Table and Its ImplementationJ.Software Journal,2004,15(3):360.

[17] He Liang, Gu Yu, Pan Jianping et al. On-demand Charging in Wireless Sensor Networks: Theories and Applications: Mobile Ad-Hoc and Sensor Systems (MASS), 2013 IEEE 10th International Conference on,2013[C]. Hangzhou: IEEE,2013: 28-36.

[18] Payam Naghshtabrizi, Joao P. Hespanha. Wireless Networking Based Control[M]. New York: Springer New York,2011:1.

[19] Caifeng Zou, Jiafu Wan, Min Chen et al. Embedded and Multimedia Computing Technology and Service[M]. Netherlands: Springer Netherlands,2012:269. 